\title{
RECEIVER GAIN CALIBRATION FOR RADIO OBSERVATIONS AT WASEDA NASU PULSAR OBSERVATORY
}

\author{
K. Niinuma ${ }^{1,2}$, M. Kuniyoshi ${ }^{3}$, N. Matsumura ${ }^{3}$, K. Takefuji $^{1}$, S. Kida $^{1}$, A. Takeuchi $^{1}$, \\ R. Nakamura ${ }^{1}$, S. Suzuki ${ }^{1}$, H. Ichikwa ${ }^{5}$, K. Asuma ${ }^{3,4}$ and T. Daishido ${ }^{2}$
}

\begin{abstract}
We have started programs for identifying EGRET $\gamma$-ray sources and searching for radio transients at the Waseda Nasu Pulsar Observatory. The observed frequency was $1.4 \pm 0.01 \mathrm{GHz}$. Eight-element spherical dish antennas were used as four pairs of interferometers, each element $20 \mathrm{~m}$ in diameter. The observable region was $\pm 5^{\circ}$ from zenith, covering a region of sky $32^{\circ} \leq \delta \leq 42^{\circ}$ in declination. For accurate flux measurement, we developed a receiver-gain calibration system using Johnson-Nyquist noise; the system was compact, inexpensive, and easily maintained. We describe the system and recent results of radio source monitoring.
\end{abstract}

Subject headings: Instrumentation: interferometers- Methods: gain calibrationTechniques: Johnson-Nyquist noise

\section{INTRODUCTION}

Since the launch of the Compton Gamma-Ray Observatory (CGRO) on April 5 1991, $271 \gamma$-ray sources were found by the Energetic Gamma-Ray Experiment Telescope (EGRET)

\footnotetext{
${ }^{1}$ Astrophysics, Department of Pure and Applied Physics, Graduate School of Science and Engineering, Waseda University, 3-4-1 Okubo, Shinjuku-ku, Tokyo 169-8555

${ }^{2}$ Department of Science, School of Education, Waseda University, 1-6-1 Nishi-waseda, Shinjuku-ku, Tokyo $169-8050$

${ }^{3}$ Institute for Astrophysics, Project Research Institutes Comprehensive Research Organization, Waseda University, 3-4-1 Okubo, Shinjuku-ku, Tokyo 169-8555

${ }^{4}$ Kuki High School, 3-12-1 Honmachi, Kuki City, Saitama 346-0005

${ }^{5}$ Kyocera Corporation, Kagahara 2-1-1, Tsuzuki-ku, Yokohama, Kanagawa 224-0055
} 
during its 10 years of observations. These sources are listed in the $3^{\text {rd }}$ EGRET Catalogue. More than half of the sources have not been identified yet, because of the large error box of $2^{\circ}$ by $2^{\circ}$. In addition, several astronomers have been carried out the effort for radio identification of EGRET unidentified $\gamma$-ray sources by comparing with known radio catalogs which are Green Bank 6cm, 21cm Survey (Gregory et al. (1996); White \& Becker (1992)) or NVSS mainly (Emmerd et al. 2003). Some of the identified sources at high Galactic latitude are blazars, and identified sources in the Galactic plane are $\gamma$-ray/radio pulsars or radio-loud micro-quasars like GRO1655-44.

Surveys of the Galactic plane were already carried out repeatedly to search for radio transients and variable radio sources at with Green Bank 90-m telescope at $4.76 \mathrm{GHz}$ (bandwidth is $580 \mathrm{MHz}$ and the HPBW is $2^{\prime} .7$ ) by Gregory \& Taylor $(1981,1986)$. However the region we could observe included high Galactic latitudes as well as the Galactic plane (Daishido et al. (1996, 2000); Takeuchi et al. (2005)). Since April 2004, at the Waseda Nasu Pulsar Observatory (see Figure 1), we have been monitoring a region of the sky $32^{\circ} \leq \delta \leq 42^{\circ}$ to find radio variable sources, which may include transient radio sources or counterparts of unidentified EGRET $\gamma$-ray sources. There are 13 unidentified EGRET sources in our observing regions.

The data of four fringe beams were obtained simultaneously, since we made observations with four pairs of two-element interferometers using drift-scanning and phase-switching (Kuniyoshi et al. 2006) at $1.4 \mathrm{GHz}$. The bandwidth of the receiver is $20 \mathrm{MHz}$, however the time resolution is 0.01 second since received signals, passed through the low-pass filter, were recorded on hard disk and digitized with an analog to digital converter (ADC) sampling at $100 \mathrm{~Hz}$. The transit time is approximately 4 minutes since the half-power beam width (HPBW) is $0.8^{\circ}$. Since each beam covers a different $0.8^{\circ}$ in declination, a region of $0.8^{\circ} \times 4$ (the field of view $\left.(\mathrm{FOV})=3.2^{\circ}\right)$ is covered in one observing session. These sessions were carried out continuously for two or three weeks.

We can observe from $0 \mathrm{~h}$ to $24 \mathrm{~h}$ in R.A. on the same declination line in a day because of drift-scan observations for wide-field survey. So, if there is a radio counterpart to unidentified EGRET source in observing declination, we can observe this source during two or three weeks continuously since we have carried out scheduled observation. One cycle of our sky survey program will be completed in approximately four months and we will repeat this program. Recently, the third survey program was completed. So, the same source will be re-observed at four month-intervals and we quantify its variability from day-intervals to month-intervals. We will continue the radio identification program of observable EGRET unidentified sources until all of these sources will be identified at radio wavelength in parallel with the program of search for radio variable and radio transient sources. 
In this paper, we describe the receiver system in Section 2, and we discuss JohnsonNyquist noise in Section 3 and the gain calibration method in Section 4. In Section 5, we describe the performance of the gain calibration system and present results. We conclude our report in Section 6

\section{RECEIVER GAIN CALIBRATION AND RADIO SOURCE VARIABILITY}

Low-noise High Electron Mobility Transistor (HEMT) receivers were used at the first stage of a heterodyne system in the Nasu Pulsar Observatory. Receiver noise temperatures were measured by Ichikawa et al. (2004) to be $40 \mathrm{~K}$ at an ambient temperature of $290 \mathrm{~K}$ and $20 K$ at liquid Nitrogen temperature of $77 K$.

The receiver gains also changed with temperature; for example, the gain was higher at low temperature. Since our receivers were not cooled, it was necessary to precisely measure the temperature dependence of the receiver gain to accurately determine the flux density of the radio sources.

Figure 2 shows raw data from a two-element interferometer for a 24-h observation period of the sky at a declination of $\delta=41^{\circ}$. The strong sources Cygnus A and Cygnus X regions in our Galaxy are seen around 6:00 Japan Standard Time (JST).

For the gain calibration, we fed Johnson-Nyquist noise to the 1st-stage HEMT amplifiers via terminating resistors. In Figure 2, the calibration signals present every thirty minutes are shown on the observed sky intensity (details are discussed in Section 4). The calibration signals became slightly weaker after sunrise at about 6:00, which was interpreted as being caused by a reduction in receiver gain at the higher temperatures.

The relationship between the ambient temperature of the receivers, $T$, and their output power, $P$, is shown in Figure 3. We empirically obtained the linear equation $P=$ $-0.0227 T+10.978$, showing a negative coefficient for the receiver gain variation versus receiver temperature; here, $T$ is the receiver temperature and $P$ is the output power of the terminated receiver in arbitrary units. 


\section{JOHNSON-NYQUIST NOISE AS A STANDARD SOURCE FOR RECEIVER NOISE MEASUREMENT AND GAIN CALIBRATION}

Planck's law of black body radiation is described by the Rayleigh-Jeans formula at low frequencies, which is derived from a statistical consideration of radiation fields interacting with matter. Therefore, we must consider the fluctuations when we measure very weak power in the microwave region. Johnson-Nyquist noise is the output voltage fluctuations of the terminating resistor. Electrons and the lattice in the resistor are in thermal equilibrium.

The ensemble mean power of the lattice, which is proportional to temperature $T$, is also equal to the Johnson-Nyquist noise power. Voltage fluctuations in the Johnson-Nyquist noise are described by $<V^{2}>=4 k T R \Delta f$, which shows that the power of thermal radiation fields is proportional to the temperature of the lattice $T$ and also to the efficiency of interactions $R$ (=resistance). For example, in a resistor having large $R$, electrons frequently exchange their kinetic energies with vibration energies of the lattice.

It should be noted that thermodynamic theory demands that the available power be half of the noise power. The above equation means that Johnson-Nyquist noise can be regarded as an adequate standard source for measuring the noise temperature and gain of the receivers, if we know the temperature $T$ of the terminating resistor. We describe our gain calibration system based on this concept in next section.

\section{RECEIVER-GAIN CALIBRATION SYSTEM IN NASU PULSAR OBSERVATORY}

In this section, the method of gain calibration used in the Nasu Pulsar Observatory is described using simulation data for two calibration signals of the terminating resistor.

We sampled the output power of the terminating resistors by disconnecting the HEMT amplifiers from the sky data and connecting them to the terminating resistors for several tens of seconds, every 30 minutes (see Figure 4).

\subsection{Calculating Receiver Gain and Linear Interpolation}

The simulation data is shown schematically in Figure 5.

The receiver gain is calculated by using the sky power (background radiation $3 K$ ) as a data offset and the output power of the terminating resistor as Johnson-Nyquist noise power 
at ambient temperature. The temperature around the terminating resistor is monitored once every 30 minutes. The data output is related to the receiver gain and system noise temperature $\left(T_{\text {sys }}\right)$ by the following equations:

$$
\begin{aligned}
Z_{1} & =G_{l i n}\left(T_{1}\right)\left(T_{1}+T_{\text {sys }}\right) \\
X_{1} & =G_{l i n}\left(T_{1}\right)\left(T_{3 K}+T_{\text {sys }}\right) \\
Z_{2} & =G_{l i n}\left(T_{2}\right)\left(T_{2}+T_{\text {sys }}\right) \\
X_{2} & =G_{l i n}\left(T_{2}\right)\left(T_{3 K}+T_{\text {sys }}\right)
\end{aligned}
$$

From (1) and (2), we obtain:

$$
\begin{aligned}
Z_{1}-X_{1} & =G_{l i n}\left(T_{1}\right)\left(T_{1}-T_{3 K}\right) \\
\Longleftrightarrow G_{l i n}\left(T_{1}\right) & =\frac{Z_{1}-X_{1}}{T_{1}-T_{3 K}}
\end{aligned}
$$

In a similar way, from (3) and (4), we obtain:

$$
\begin{aligned}
Z_{2}-X_{2} & =G_{l i n}\left(T_{2}\right)\left(T_{2}-T_{3 K}\right) \\
\Longleftrightarrow G_{l i n}\left(T_{2}\right) & =\frac{Z_{2}-X_{2}}{T_{2}-T_{3 K}}
\end{aligned}
$$

Thus, the receiver gain $G_{l i n}\left(T_{1}\right)$ and $G_{l i n}\left(T_{2}\right)$ is calculated at ambient temperatures $T_{1}$ and $T_{2}$.

Additionally, by substituting (5) into (1), we can also calculate $T_{\text {sys }}$ as follows:

$$
T_{\text {sys }}=\frac{Z_{1}}{G_{l i n}\left(T_{1}\right)}-T_{1}=\frac{X_{1} T_{1}-Z_{1} T_{3 K}}{Z_{1}-X_{1}}
$$

Therefore, it is possible to calculate the gradient of the receiver gain $\left(\Delta G_{\text {lin }}(T) / \Delta t\right)$ between time $t_{1}$ and time $t_{2}$, and to divide the data between $t_{1}$ and $t_{2}$ by $\Delta G_{l i n}(T) / \Delta t$. As a result, the term $\left(T+T_{\text {sys }}\right)$ is obtained each time because the fluctuation of the receiver gain is calibrated between $t_{1}$ and $t_{2}$. If $T_{\text {sys }}$ is subtracted from $\left(T+T_{s y s}\right)$, only the output power of the source antenna temperature is obtained.

First, the gradient of the receiver gain has to be calculated between times $t_{1}$ and $t_{2}$ as follows:

$$
\frac{\Delta G_{l i n}(T)}{\Delta t}=\frac{G_{l i n}\left(T_{2}\right)-G_{l i n}\left(T_{1}\right)}{t_{2}-t_{1}}
$$

Next, the receiver gain between $t_{1}$ and $t_{2}$ is removed by using (8):

$$
\operatorname{data}_{1}(t)=\operatorname{data}_{0}(t) /\left(\frac{\Delta G_{l i n}(T)}{\Delta t} t+G_{l i n}\left(T_{1}\right)\right)
$$


where $\operatorname{data}_{0}(t)$ is without receiver gain calibration and $d a t a_{1}(t)$ is with receiver gain calibration.

For example, if the data between $t_{1}$ and $t_{2}\left(T_{1}\right.$ and $\left.T_{2}\right)$ includes source signals of temperature $T_{s}$, it is possible to obtain the output power which corresponds to the source temperature by correcting for the fluctuation of the receiver gain and $T_{\text {sys }}$.

\subsection{Observed Data with Gain Calibration}

We applied the above calibration method to the data observed in the Nasu Pulsar Observatory.

If our observed data is detected without using a square-law detector, the signal is represented by a sine wave whose amplitude increases in proportion to the input temperature. That is, it is possible to calculate the receiver gain and $T_{\text {sys }}$ by measuring the amplitude at two different temperatures (Ichikawa et al. 2004).

However, our observed data was obtained as a direct current because the data was detected with a square-law detector. Therefore, it was difficult to measure the offset $T_{\text {sys }}$ which is added to the data.

Nevertheless, we could obtain the difference between the output power of the terminating resistor at ambient temperature and that of the sky (cosmic background radiation at $3 K$ ), in other words the value of $(Z-X)$, to calculate the receiver gain momentarily. As a result, it was possible to correct for fluctuations of the receiver gain due to the temperature dependence. In Figure 6, we show the daily fringes of Cygnus A with and without gain calibration. In Figure 7, the top is a map of the region around Cygnus A observed at the Waseda Nasu Pulsar Observatory, and the bottom is the contour map. These are a part of the first map using this gain calibration system for our observatory. We showed W58 (HШ region), B1922 $+3323(4.0 \mathrm{Jy} \text { at } 1.4 \mathrm{GHz} \text {, spectral index }=-0.6 \text { at } 1.4-5 \mathrm{GHz})^{1}$ and new radio source as the examples of observable radio sources at our observatory by marking up. We found new radio source (WJN J1954+3600) at $\alpha=19^{h} 54^{m} 35^{s} \pm 10^{s}$ on the $\delta=36^{\circ}$ line. This source appeared every day in our observations from December 282004 to January 232005. The flux density of this source is approximately $2 \mathrm{Jy}$. However such a strong radio source, which is over $1 \mathrm{Jy}$ at $1.4 \mathrm{GHz}$, was not listed in $1420 \mathrm{MHz}$ northern sky survey catalogue ${ }^{1}$ or

\footnotetext{
${ }^{1}$ White \& Becker (1992)
} 
NVSS catalogue ${ }^{2}$

\section{EXPERIMENTAL RESULTS IN ACCURATE FLUX MEASUREMENT}

In this section, we evaluate the results of the receiver gain calibration using JohnsonNyquist noise.

The radio sources $3 \mathrm{C} 41(3.5 \mathrm{Jy})^{1}$, 3C48 (15.8 Jy $)^{1}$, 3C84 (21.2 Jy) ${ }^{1}$, 3C119 (7.5 Jy $)^{1}$, B0415+3754 (13.5 Jy) ${ }^{1}$, B0501+3801 (8.9 Jy) ${ }^{1}$, MG2001+3332 (12.8 Jy at 5GHz, spectral index $=0.02$ at $1.4-5 \mathrm{GHz})^{3}, \mathrm{~B} 1611+3420(2.9 \mathrm{Jy})^{4}$, and Cygnus A $(1590.0 \mathrm{Jy})^{2}$ were used to evaluate our gain calibration system. These sources were observable at $1.4 \mathrm{GHz}$ in the Nasu Pulsar Observatory.

There are two reasons that these sources were chosen for evaluating the system. First, they were detectable at ten or more times the detection limit. Second, their spectral energy distribution (SED) decreased from low frequency to high frequency, and there was little or slight long-term variability, according to the NASA/IPAC Extragalactic Database. In addition, B1611+3420 showed a flat spectrum and variability similar to BL Lac at radio frequencies, and this source was already identified as the EGRET $\gamma$-ray source 3EG $\mathrm{J} 1614+3424$. We added B1611+3420 to the target radio sources, since we had to evaluate whether there was a difference between using a steady radio source and a variable radio source as the target source in our system.

The receiver gain calibration system was evaluated by conducting the following three comparisons.

- Comparing (1) the correlation between a target source and a suitable radio source serving as a standard source near the target with (2) the correlation between a target source and Johnson-Nyquist noise serving as a standard source near the target. Here, suitable radio source means a source having steady or slightly long-term variability and strong flux density at radio frequencies, such as 3C48 or 3C84.

- Comparing the estimated fluctuations (EF) of flux density with and without receiver

\footnotetext{
${ }^{2}$ NASA/IPAC EXTRAGALACTIC DATABASE, http://nedwww.ipac.caltech.edu/

${ }^{3}$ Griffith et al. (1990)

${ }^{4}$ This source is radio loud flat spectrum radio quasar (FSRQ), and counterpart of identified EGRET $\gamma$-ray source 3EG J1614+3424 (Mattox et al. 2001)
} 
gain calibration for several sources. We defined EF as the following equation.

$$
E F=\sqrt{\frac{1}{N} \sum_{i=1}^{N} F_{i}^{2}-\left(\frac{1}{N} \sum_{i=1}^{N} F_{i}\right)^{2}} / \frac{1}{N} \sum_{i=1}^{N} F_{i}
$$

where, $F_{i}$ is the daily flux densities of radio sources, $N$ is the data number.

- Comparing EF of fringe amplitude with and without receiver gain calibration by using Johnson-Nyquist noise or a suitable radio source as a standard source.

These are described in turn in the following subsections.

\subsection{Comparing Correlation between Target Source and Standard Source}

We compared two kinds of correlation without receiver gain calibration to show that the gain calibration using Johnson-Nyquist noise was effective in achieving accurate flux measurement. One was the correlation between target source 3C41 and standard source 3C48 near 3C41; and the other was the correlation between 3C41 and the output power of the terminating resistor serving as a standard source near 3C41.

If target and standard sources exhibited a strong correlation, it would indicate that each signal was detected with the same receiver conditions (gain, $T_{\text {sys }}$, etc.).

Figure 8 show that using the output power of the terminating resistor as the standard source resulted in a higher correlation than using 3C48. Therefore, using the output power of the terminating resistor is effective for high-accuracy flux measurement.

\subsection{Comparing Flux Density with and without Gain Calibration}

In this section, EF of the flux density of MG2001+3332, B0415+3754 (3C111), and B0501+3801 (3C134) were compared with and without receiver gain calibration. The flux density of these sources was calculated by the following method:

- We calculated the antenna temperature for the output power of the terminating resistor and Cygnus A by using their amplitudes and the monitored ambient temperature.

- We calculated the relation between the antenna temperature and the flux density of Cygnus A (1590 Jy at 1.4GHz; given parameter). 
- Using the relation between the antenna temperature and the flux density calculated above, we calculated the flux densities of MG2001+3332, 3C111, and 3C134 by comparing the antenna temperatures for the output powers of the terminating resistor with those of these sources.

As a result, the flux density with gain calibration exhibited smaller fluctuations and flatter light curves than without gain calibration (see Figure 9 and Table. 2).

\subsection{Comparing Two Standard Sources: Johnson-Nyquist Noise and Suitable Radio Source}

Finally, EF of the fringe amplitude were compared for several sources having high signalto-noise ratio.

As seen in Figure 9 described above, we compared data with gain calibration to data without gain calibration by using the output power of the terminating resistor.

However, in order to verify whether using the output power of the terminating resistor removed the fluctuations of fringe amplitude as effectively as using the suitable radio source, we had to compare the fluctuations of fringe amplitude in both cases.

In this experiment quasar 3C48 and radio galaxy 3C84 were suitable as standard sources, so $3 \mathrm{C} 41$ was calibrated with the output power of the terminating resistor and $3 \mathrm{C} 48$, and $3 \mathrm{C} 119$ was calibrated with the output power of the terminating resistor and $3 \mathrm{C} 84$. These results are shown in Table 3.

If there is little difference between the two methods, that is, using the output power of the terminating resistor and using a suitable radio source as the standard source for correcting the fluctuations in receiver gain, it is always possible to calibrate the receiver gain to allow accurate flux measurement by using the output power of the terminating resistor.

As a result, the fluctuations in the daily fringe amplitude for each radio source during each observation period were removed more effectively by using our receiver gain calibration system than by using suitable radio sources (see Table 3).

\section{DISCUSSION AND CONCLUSION}

EF in daily fringe amplitude for steady sources were improved by approximately $10 \%$ using the output power of a terminating resistor as a standard source. This means that the 
detection limit was reduced in identifying the radio variability of target sources, such as active galactic nuclei (AGN), EGRET counterparts, and transient sources. Our newly developed receiver-gain calibration system improved the measurement accuracy, and in addition, the system was compact, inexpensive, and easily maintained.

Although we developed this gain calibration system for a two-element interferometer, it should be possible to apply this method to the observations of eight-element grating interferometer of 20-m-diameter dish array at our observatory, or other grating interferometer using many more antennas such as the square kilo-meter array (SKA), that is a new generation program of radio astronomy. In that case, since we need consider only that the number of antennas is larger compared to two-element interferometer, we need rewrite a little bit more the discussed equations in section 4 as following equations. If the data will be recorded with Nyquist rate sampling, it is possible to estimate the receiver gain and $T_{\text {sys }}$ every antennas or as a grating interferometer, and to calibrate the fluctuations of those.

The output power of each signal is represented as next equations.

$$
\begin{aligned}
\sigma_{k-300 K}^{2} & =\frac{1}{\tau}\left(\sum_{i=0}^{\tau} V_{k-i}^{2}-\left(\sum_{i=0}^{\tau} V_{k-i}\right)^{2}\right) \\
\sigma_{k-3 K}^{2} & =\frac{1}{\tau}\left(\sum_{j=0}^{\tau} V_{k-j}^{2}-\left(\sum_{j=0}^{\tau} V_{k-j}\right)^{2}\right)
\end{aligned}
$$

where, $k=$ antenna number, $\sigma_{k-300 K}^{2}$ is the variance of the output power of calibration signal, $\sigma_{k-3 K}^{2}$ is the variance of sky power, $\tau$ is the duration of input calibration signal, $V_{i}$ is the data of input calibration signal, $V_{j}$ is the data of sky just before (or just after) input calibration signal.

Considering that the receiver gain of N-element grating interferometer is calculated, the following equations are obtained.

$$
\begin{aligned}
\sum_{k=1}^{N}\left(\sigma_{k-300 K}^{2}\right) & =\sum_{k=1}^{N}\left(G_{k}\left(t_{0}\right)\left(T_{k-300 K}\left(t_{0}\right)+T_{\text {sys-k }}\left(t_{0}\right)\right)\right) \\
\sum_{k=1}^{N}\left(\sigma_{k-3 K}^{2}\right) & =\sum_{k=1}^{N}\left(G_{k}\left(t_{0}\right)\left(T_{3 K}+T_{\text {sys-k }}\left(t_{0}\right)\right)\right) \\
\Longleftrightarrow G_{\text {total }}\left(t_{0}\right) & \approx \frac{\sigma_{\text {total }-300 K}^{2}-\sigma_{\text {total }-3 K}^{2}}{T_{300 K}\left(t_{0}\right)-T_{3 K}} \\
T_{\text {sys-total }}\left(t_{0}\right) & \approx \frac{\sigma_{\text {total-3K}}^{2} T_{300 K}\left(t_{0}\right)-\sigma_{\text {total }-300 K}^{2} T_{3 K}}{\sigma_{\text {total }-300 K}^{2}-\sigma_{\text {total }-3 K}^{2}}
\end{aligned}
$$

where, $G_{k}\left(t_{0}\right)$ is the receiver gain of each antennas, $G_{\text {total }}\left(t_{0}\right)$ is the receiver gain as a grating interferometer, $T_{\text {sys-k }}\left(t_{0}\right)$ is the system noise temperature of each antennas, $T_{\text {sys-total }}\left(t_{0}\right)$ is 
the system noise temperatureof as a grating interferometer, $T_{k-300 K}\left(t_{0}\right)$ is the monitored ambient temperature around the terminating resistor of each antennas, $t_{0}$ is the time calibration signal is input. And, we applied next approximation to above equations.

$$
\sum_{k=1}^{N}\left(G_{k}\left(t_{0}\right)\left(T_{k-300 K}\left(t_{0}\right)-T_{3 K}\right)\right) \approx G_{t o t a l}\left(t_{0}\right)\left(T_{300 K}\left(t_{0}\right)-T_{3 K}\right)
$$

Finally, steady radio sources and one radio-loud quasar over a short span were evaluated in this experiment. The results of using our gain calibration system indicate the possibility of rapidly identifying day- or week-scale variability, such as blazars or transient sources, when those sources appear in the observed region.

This research made use of the NASA/ IPAC Extragalactic Database (NED), which is maintained by the Jet Propulsion Laboratory, California Institute of Technology, under contract with NASA. This research also made use of data obtained from the High Energy Astrophysics Science Archive Research Center (HEASARC), supported by the NASA Goddard Space Flight Center. This research was supported by Grant-in-Aid for Scientific Research (A) 14204027, Academic Frontier Project for Private Universities with a matching subsidy from MEXT (Ministry of Education, Culture, Sports, Science and Technology), Waseda University Grant for Special Research Projects 2006A-848, and The 21st Century COE Program at Waseda University.

\section{REFERENCES}

Daishido, T., Tanaka, N., Sudo, S., et al., 1996 ASPC, 105, 19

Daishido, T., Tanaka, N., Takeuchi, H., et al., 2000 Proc. SPIE, 4015, 73

Emmerd, D. S., Romani, R. W. and Michelson, P. F., 2003, ApJ, 590, 109

Ichikawa, H., et al. 2004, Frontier in Astroparticle Physics and Cosmology (UNIVERSAL ACADEMY PRESS, INC.), 391.

Gregory, P. C., Scott, W., K., Douglas, K., 1996, ApJS, 103, 427

Gregory, P. C., Taylor, A. R., 1986, AJ, 92, 371

Gregory, P. C., Taylor, A. R., 1981, ApJ, 248, 596 
Griffith M., Langston G., Heflin M., et al., 1990, ApJS, 74, 129.

Kuniyoshi, M., Daishido, T., Asuma, K., et al., 2006, PASP, 118, 901.

Matsumura, N., Kuniyoshi, M., Takefuji, K., et al., 2006, J. Phys.: Conf. Ser. 31, 193.

Mattox, J. R., Hartmann, R. C., Reimer, O., 2001, ApJS, 135, 155.

Takeuchi, H., Kuniyoshi, M., Daishido, T., et al., 2005 PASJ, 57, 815

White, R.L., Becker, R.H., 1992, ApJS, 79, 331 


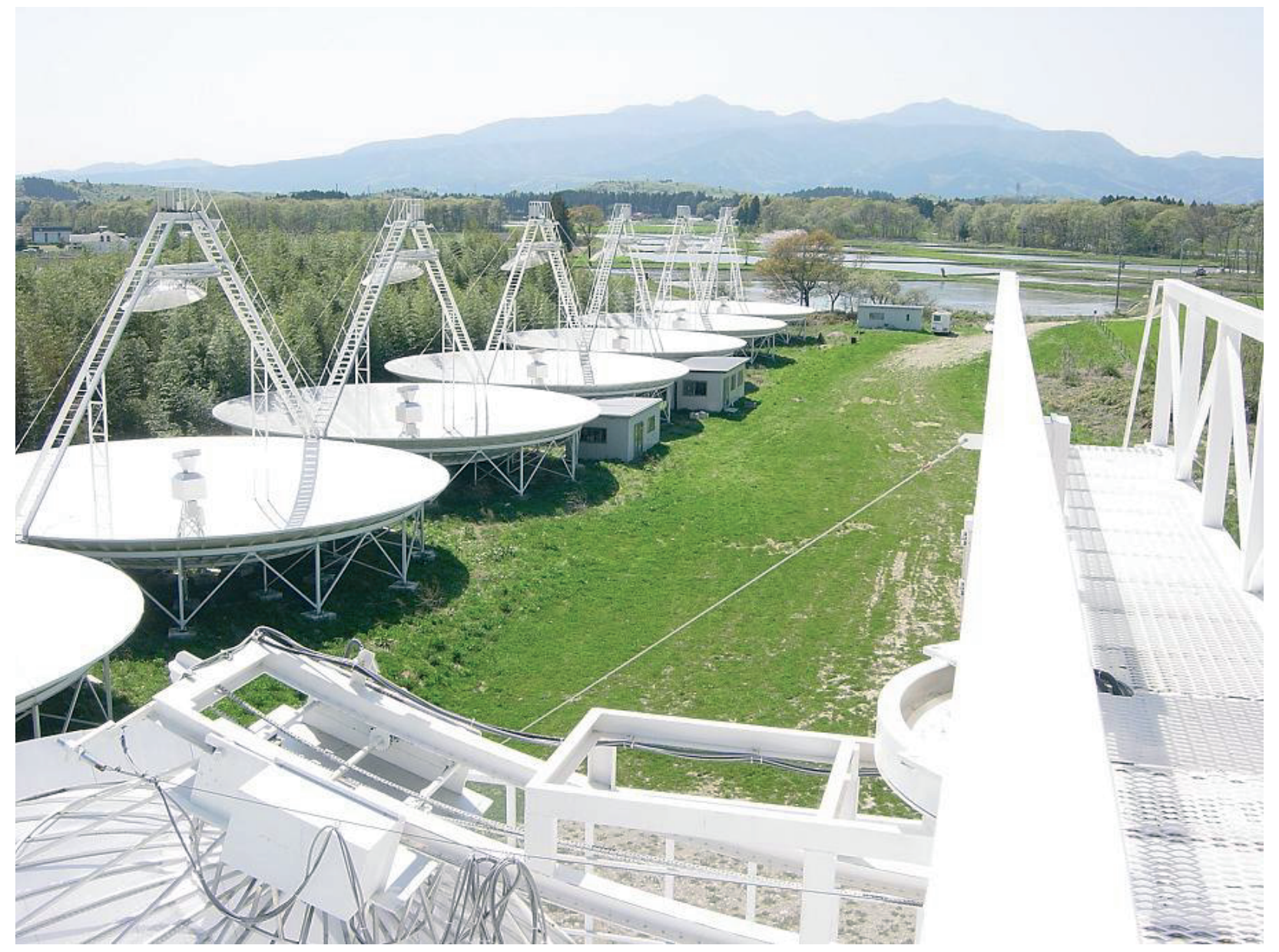

Fig. 1.- Waseda Nasu Pulsar Observatory in Nasu, Tochigi Prefecture, Japan, 160 km north of Tokyo (http://www.phys.waseda.ac.jp/astro/main-j.html). This observatory is located at latitude $36^{\circ} 55^{\prime} 41.3^{\prime \prime}$ north and longitude $139^{\circ} 58^{\prime} 54.3^{\prime \prime}$ east. The observable region of $20 \mathrm{~m}$-in-diameter dish antennas is from $32^{\circ}$ to $42^{\circ}$ in declination. This sky coverage rate is approximately $7.0 \%$ of all sky. $20 \mathrm{~m}$-in-diameter dish antennas are purely transit type, and the HPBW of each is approximately $0.8^{\circ}$. Since we have observed using four-fringe beams simultaneously for covering four different declinations, the field of view is approximately $3.2^{\circ}$. 


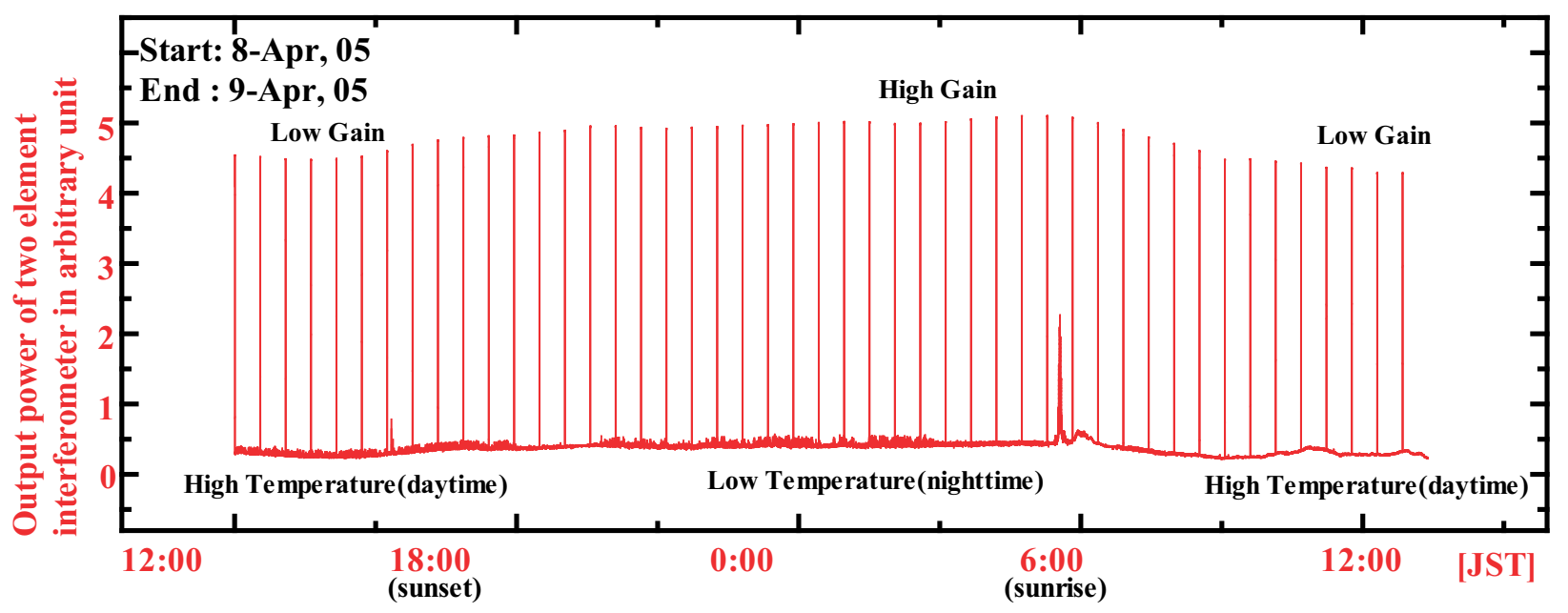

Fig. 2.- Raw data from two-element interferometer and calibration signals. Cygnus A and the Galactic plane are seen around 6:00 (JST). After sunrise at 6:00, the calibration signal levels decreased, showing the negative correlation between gain and ambient temperature.

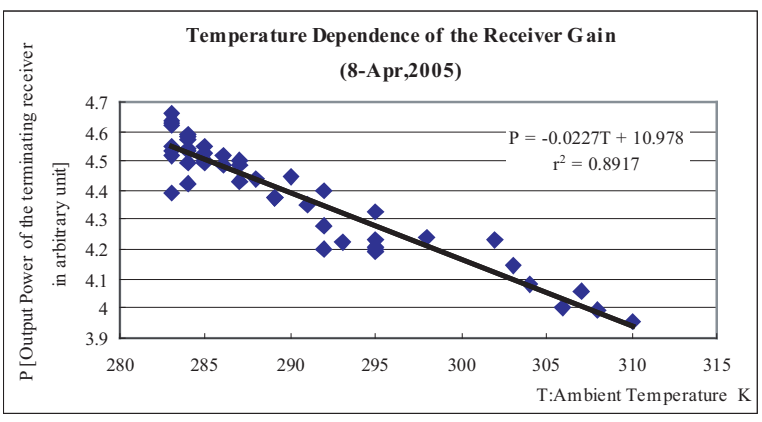

Fig. 3.- Output power of the terminated receiver at $280 K$ to $310 K$. The receiver gain was high at low temperature, and vice versa. Since the Johnson-Nyquist noise power $(\propto T)$ should increase by $10 \%$ at $310 K$ compared with that at $280 K$, this experiment shows that the gain reduction overwhelms the noise increase. Here, $r$ is the correlation coefficient. 


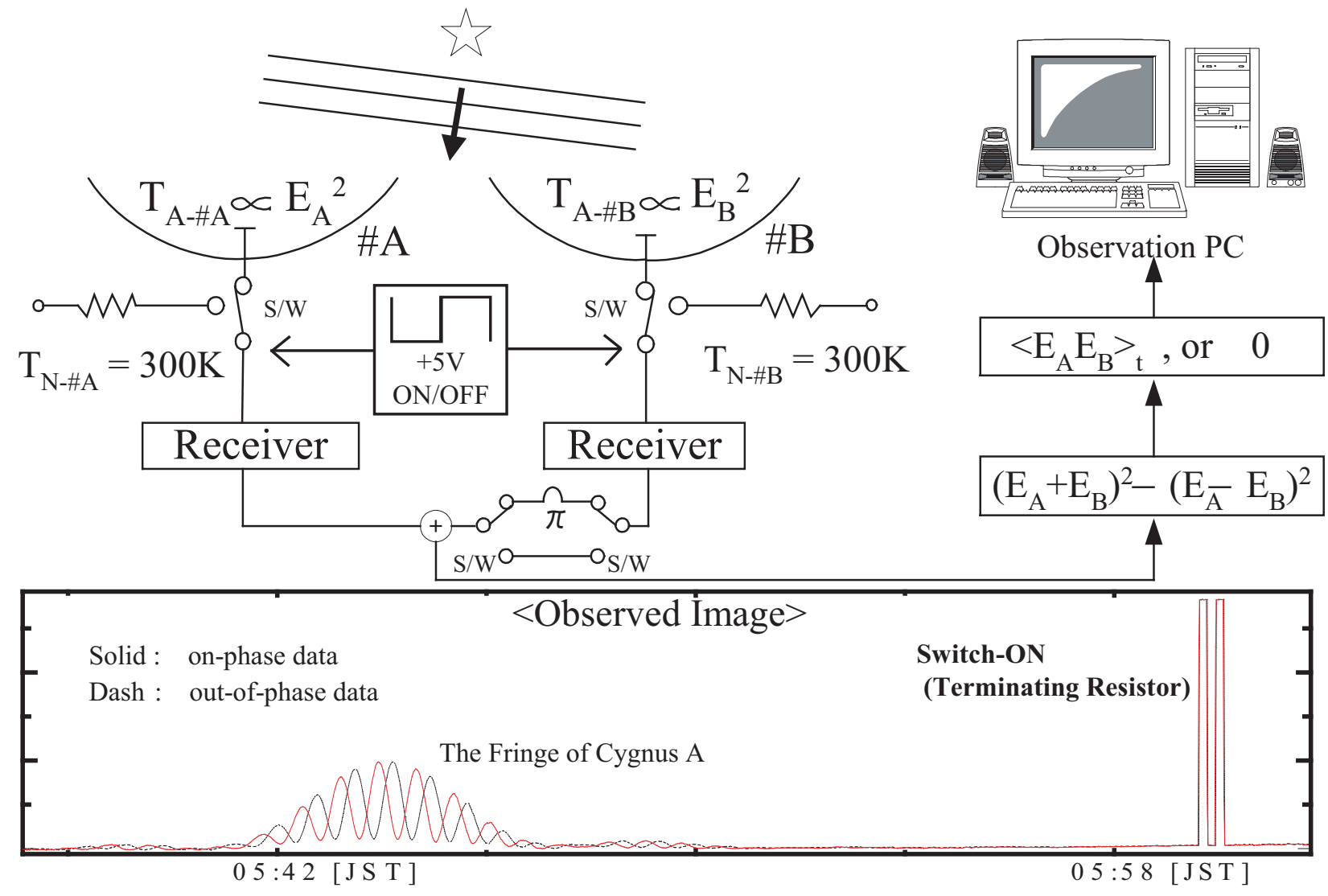

Fig. 4. - Block diagram of gain calibration system. Dummy load $\left\{<\sqrt{T_{N-\# A}} \sqrt{T_{N-\# B}}>_{t}=\right.$ 0 (no correlation) $\}$. Sky load $\left\{<E_{A} E_{B}>_{t} \neq 0\right\}$. 


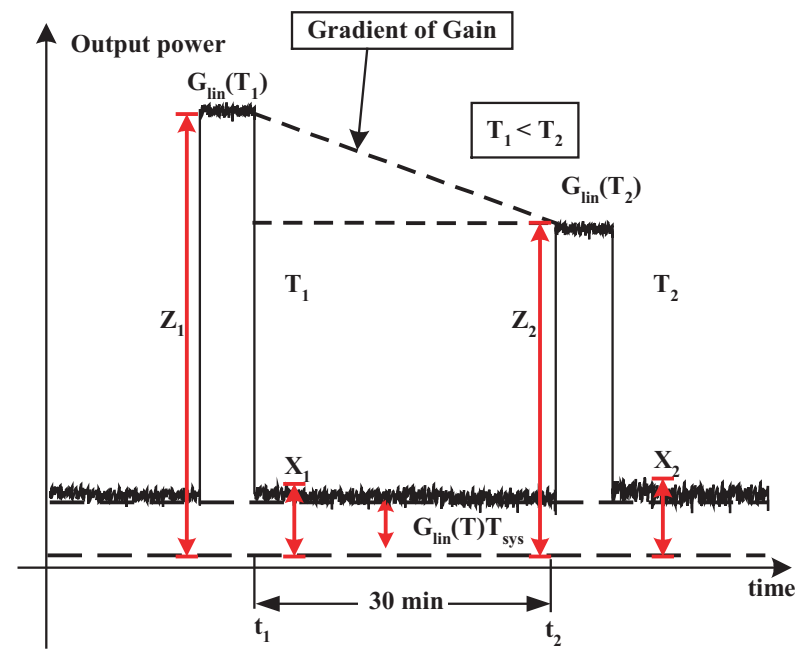

Fig. 5.- Schematic illustration (not actual data) of the output power of the terminating resistor between different ambient temperatures $T_{1}$ (at time $t_{1}$ ) and $T_{2}$ (at time $t_{2}$ ). $G_{\text {lin }}(T)$ : receiver gain (temperature dependence coefficient); $T$ : ambient temperature around terminating resistor and 1st HEMT amplifier; $Z$ : output power of terminating resistor $+T_{\text {sys }}$; and $X$ : cosmic background radiation output $+T_{\text {sys }}$.

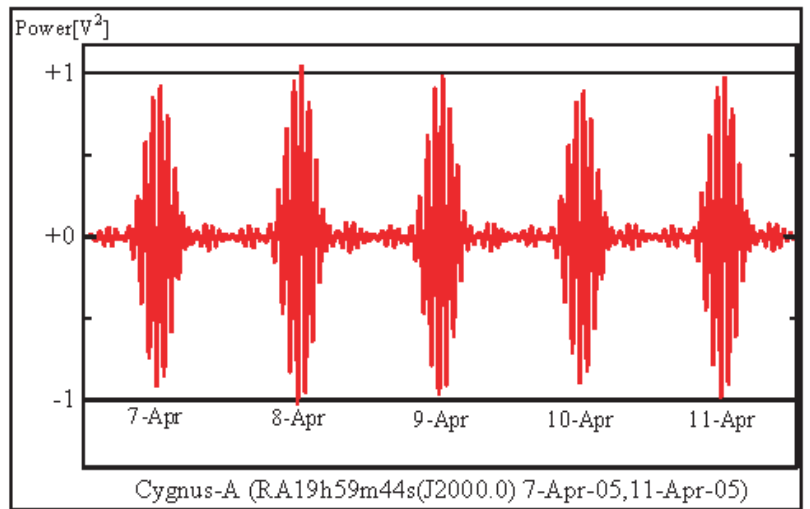

Without Gain Calibration

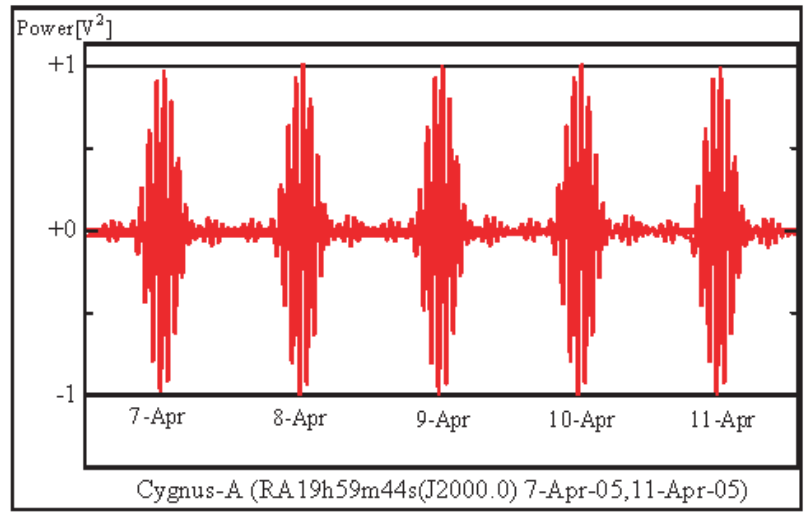

With Gain Calibration

Fig. 6.- Daily fringe data of Cygnus A. Left: without gain calibration. Right: with gain calibration. The vertical axis shows recorded value as the fringe data of Cygnus A on hard disk. The peak-to-peak of the each fringe amplitude without gain calibration doesn't line along \pm 1 of vertical scale (the fringe amplitude on Apr 7 and 10 are smaller than \pm 1 , and the one on Apr 8 is over \pm 1 ). In contrast, the peak-to-peak of the each fringe amplitude with gain calibration lines along \pm 1 of vertical scale. The estimated fluctuations, defined in section 5, from Apr 7 to 11 are $5.8 \%$ without gain calibration and $1.1 \%$ with gain calibration. 


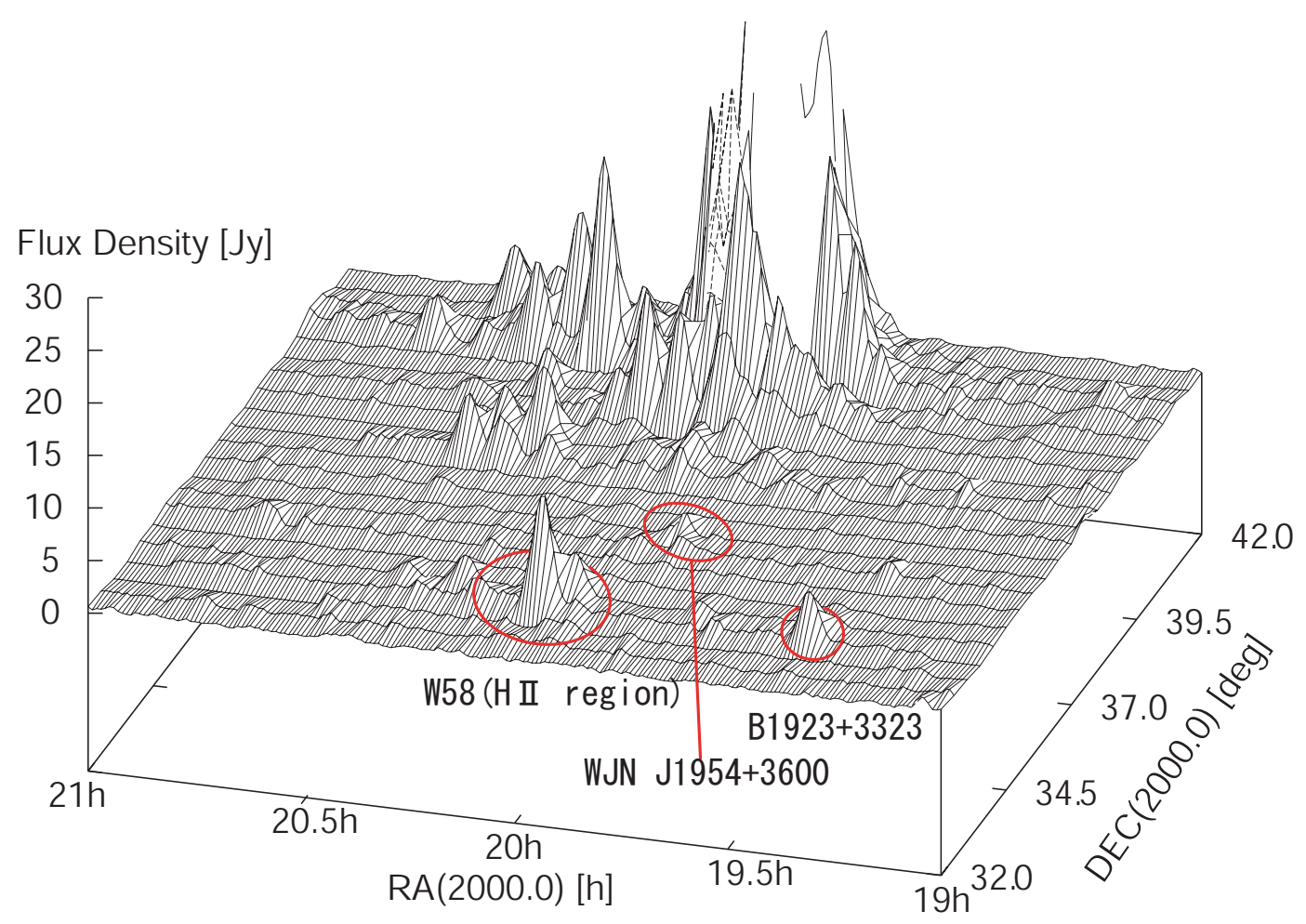

The contour interval corresponds to approximately $0.5 \mathrm{Jy}$

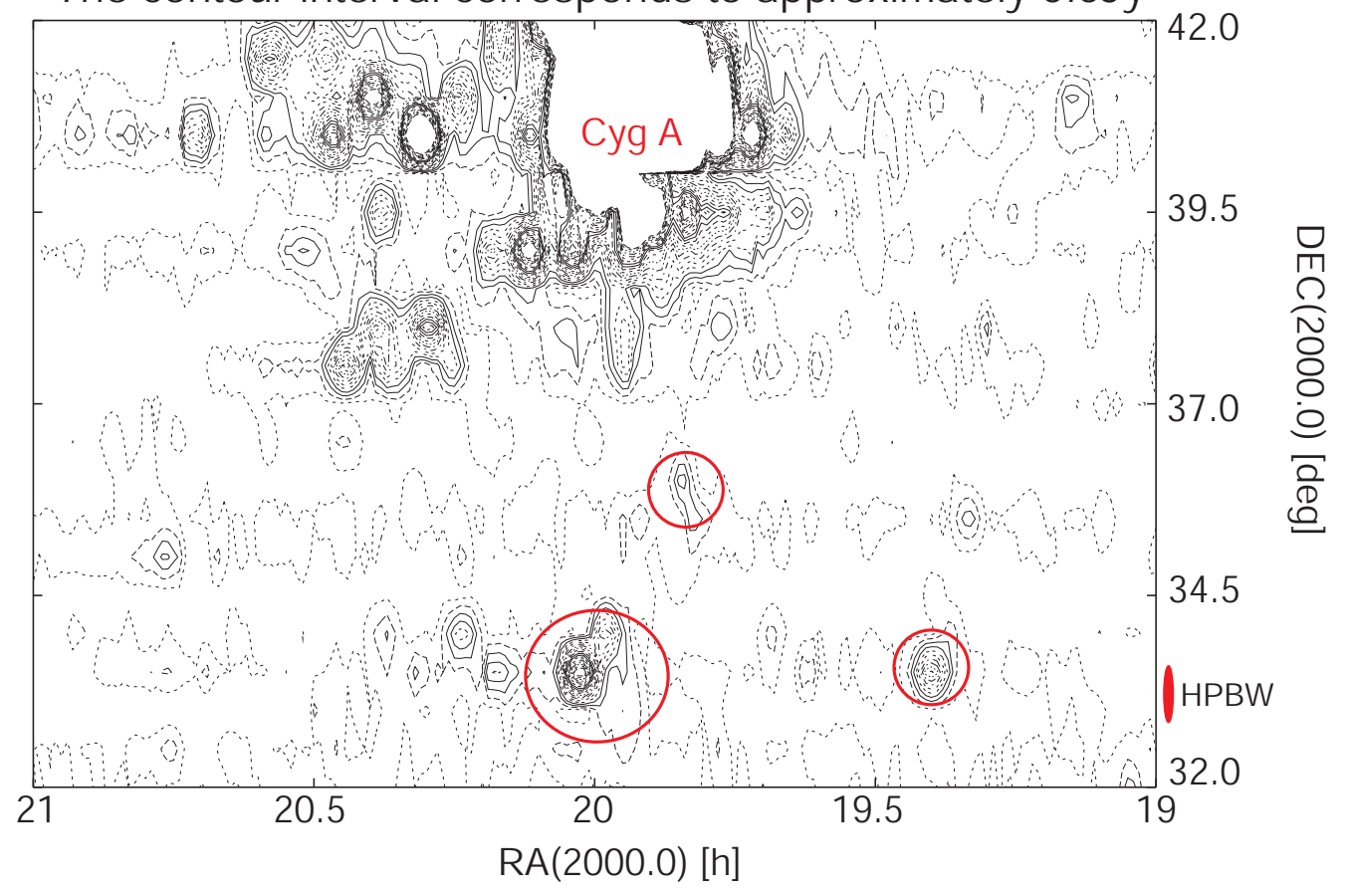

Fig. 7.- Top: Radio map around Cygnus A, detected at Waseda Nasu Pulsar Observatory. Bottom: This contour interval corresponds to approximately 0.5Jy. The Cygnus X region, W58 (left circle), and B1922+3323 (right circle) WJN J1954+3600 (center circle) can be seen. Several strong sources, including MG2001+3332 (see Section 5), are confusing in W58. 


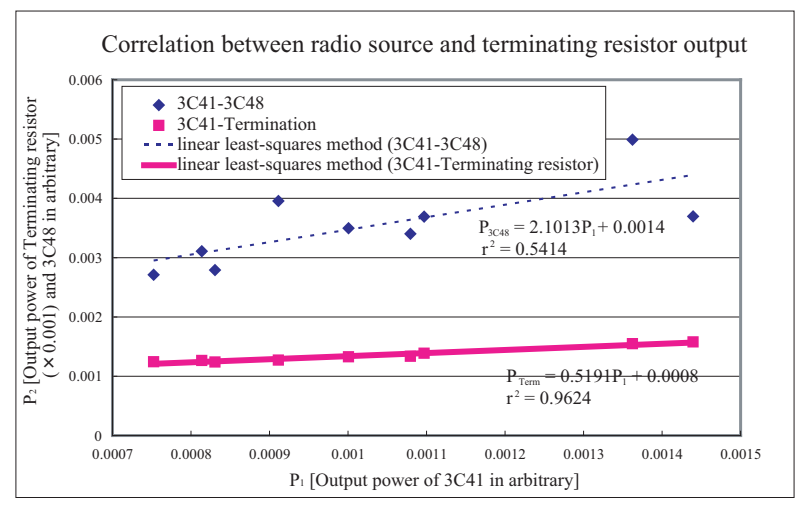

Fig. 8.- Comparing the correlation between output power of 3C41 and 3C48 with the correlation between $3 \mathrm{C} 41$ and terminating resistor. The correlation coefficient between 3C41 and the terminating resistor $\left(r^{2}=0.9624\right)$ was higher than that between $3 \mathrm{C} 41$ and $3 \mathrm{C} 48$ $\left(r^{2}=0.5414\right)$. 

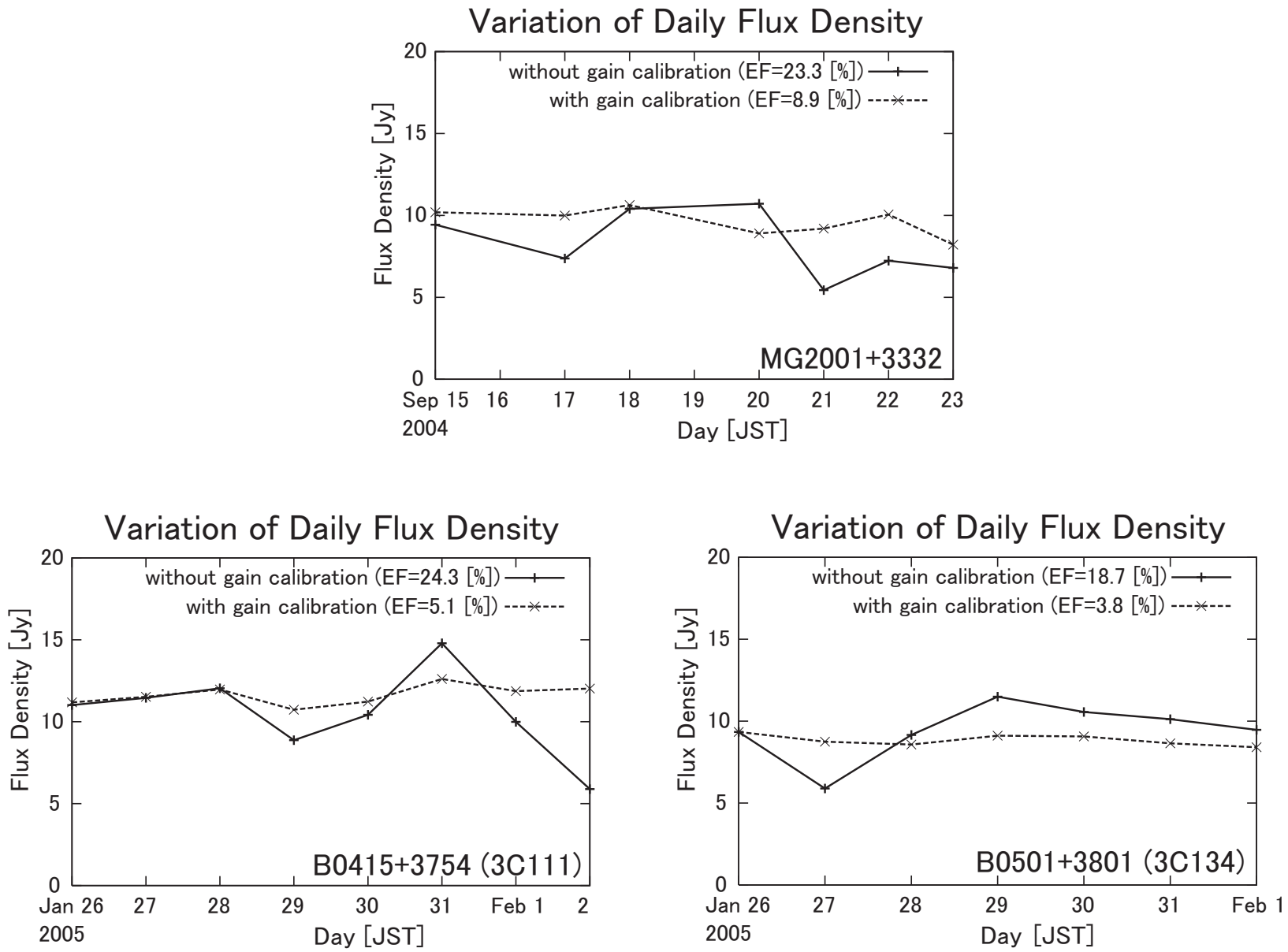

Fig. 9.- Variation of daily flux density in MG2001+3332, B0415+3754 (3C111) and B0501+3801 (3C134) with and without gain calibration. 
Table 1: Correlation coefficient between standard source 3C48 and target source 3C41 was compared with correlation coefficient between terminating resistor near 3C41 and 3C41.

\begin{tabular}{ccc}
\hline \hline Correlation Pattern & \multicolumn{2}{c}{ Correlation } \\
& Coefficient $(=r)$ \\
& $r$ & $r^{2}$ \\
\hline 3C41-3C48 & 0.7358 & 0.5414 \\
3C41-Termination & 0.9810 & 0.9624 \\
\hline
\end{tabular}

Table 2: EF in flux density with and without gain calibration

\begin{tabular}{cccc}
\hline \hline Object & $\begin{array}{c}\text { Without } \\
{[\%]}\end{array}$ & $\begin{array}{c}\text { With } \\
{[\%]}\end{array}$ & Observational Schedule \\
\hline MG2001+3332 & 23.3 & 8.9 & 2004 Sep 15-2004 Sep 23 \\
B0415+3754 (3C111) & 24.3 & 5.1 & 2005 Jan 26-2005 Feb 02 \\
B0501+3801 (3C134) & 18.7 & 3.8 & 2005 Jan 26-2005 Feb 01 \\
\hline
\end{tabular}


Table 3: EF in fringe data with and without gain calibration

\begin{tabular}{|c|c|c|c|}
\hline Object Name & $\begin{array}{c}\text { Without } \\
{[\%]}\end{array}$ & $\begin{array}{c}\text { With (terminating resistor) } \\
{[\%]} \\
\text { With (Standard source) } \\
{[\%]}\end{array}$ & Observational Schedule \\
\hline $3 \mathrm{C} 41$ & 23.3 & $\begin{array}{c}9.6 \\
15.7(3 \mathrm{C} 48)\end{array}$ & 2004 Sep 15-2004 Sep 23 \\
\hline $3 \mathrm{C} 48$ & 19.4 & $\begin{array}{c}12.2 \\
-\end{array}$ & 2004 Sep 15-2004 Sep 23 \\
\hline $3 \mathrm{C} 84$ & 11.0 & $\begin{array}{c}9.5 \\
-\end{array}$ & 2004 Dec 28-2005 Jan 23 \\
\hline $3 \mathrm{C} 119$ & 23.7 & $\begin{array}{c}15.1 \\
20.6(3 \mathrm{C} 84)\end{array}$ & 2004 Dec 28-2005 Jan 23 \\
\hline $\mathrm{B} 1611+3420$ & 44.2 & $\begin{array}{c}49.5 \\
-\end{array}$ & 2005 Jan 26-2005 Feb 02 \\
\hline Cygnus A & 7.2 & $\begin{array}{c}5.5 \\
-\end{array}$ & 2005 Apr 01-2005 Apr 16 \\
\hline
\end{tabular}

